\title{
SLEEP CONTROL FOR LIFE TIME MAXIMIZATION AND ROUTING IN WSN
}

\author{
V.Amsa ${ }^{1}$ and S. Raj Anand ${ }^{2}$ \\ ${ }^{1}$ PG.Scholar, Departement of MCA, Veltech High Tech Engineering College \\ ${ }^{2}$ Assistant Professor, Departement of MCA, Veltech High Tech Engineering College
}

\begin{abstract}
Recently the research is about the life time maximization and the routing in wireless sensor network it is an important issue in today's WSN.To solve these types of problems some of the algorithms and techniques are addressed in this paper and also introduced network architecture. To save the life time in the network.This paper proposed sleep control for the sensor node in the network and connected dominating set based alpha minimum routing cost algorithm for the routing. Wsn is a node which is connected to several or thousands of the sensor in wireless area such as radio transceiver either internal antenna or as external antenna.
\end{abstract}

\section{Keywords}

WSN, Sleep Control, Routing, Alpha Minimum Routing.

\section{Introduction}

Wireless sensor network deployed thousands of sensor nodes. Nodes are based the on batteries in WSN. To extend the life time of the battery sleep control is used and also saves the power consumption of the nodes. Sleep control uses the semi active state for all the nodes in WSN. To forward the packets or any kind of data we use routing. Routing has many kind of techniques. In this paper we use alpha minimum routing algorithm and for connection with nodes we deployed connected dominating set based. Connected dominating is set based for the nodes with its neighborhood. The main advantage is recalculation is not needed for the routing. Our proposal is focused on the routing and life time maximization in WSN. Semi active state is deployed for sleep control in order to send the data instead of sleep or wake up the nodes when the input is received. For all nodes in the network. First check the nodes whether it is in active state or it is in off state. To check this purpose we introduced connected domination set based algorithm to connect the neighbor nodes. When the connection is established, all the nodes in the network applied with semi active state and it produces the active state when the input is received. [1]Any cast packet is done using sleep-wake scheduling and it is used to reduce the wastage of energy. [2]GPSR provides per router than the shortest path and it finds the correct new router and it compares the mobile wireless sensor network with dynamic source routing. [3] GDSTR algorithm is used to find the shortest path than geographic face algorithm.[4] LCR provides the efficient the routing in WSN. [5] compared with geographic packet forwarding,CAGIF consumes much more energy and decreases the signal over heads. [6]The unsafe area can be shown in the form of rectangular region and achieve the better performances in routing. [7]EBGR provides the node based on the energy level and it finds the next node relay position. [8] Coverage of the network is done using low duty-cycled sensor random and coordinates sleep algorithm. [9]DSDA collects 
International Journal of Control, Automation, Communication and Systems (IJCACS), Vol.1, No.1, January 2016

the information from its neighbor node. [10] Efficient mobile sensor node, griddeploymentidentifies the weight of the each grid such as predeployed node, boundaries etc. Hence the main objective is to save the life time of the sensor nodes and to provide the routing with low cost, with this we can avoid the data delay and to avoid the failure nodes in the network. The need of the project is to save the energy while the transmission takes place in the WSN by sleep control and to avoid datadelay alpha minimum routing algorithm is proposed.

\section{Existing Lifetime Imrovements In Wsn}

Wireless sensor network consists of many techniques and algorithm. The existing system focus on packet forwarding and the lifetime maximization using sleep and wake up the nodes due to this proposal packets are delayed .The sleep and wake up is provided to nodes through which data reaches its destination and its has no alternative path when the node is failed. This kind of problems raised in the existing .Later a new proposal is produced Backbone algorithm. Backbone algorithm provides the routing in the limited manner to save the life time of the sensor. It does not affect the network and it saves the energy. To overcome all these problems we proposed some algorithms and techniques in this paper.

\subsection{Draw Backs Of Life Time Improvements In Wsn}

The disadvantages of the existing are power consumption, data delay, and routings.

\section{Proposed Methodolgy Of Sleep Control For Life Time Maximization In Wsn}

The proposal of this paper concentrates in life time maximization, routing and sleep control in WSN.Semi active state is applicable for the nodes in the network, which provides semi active strategies. The strategies include gain scheduling and clipped optimal schemes with continuouslyvarying and on-off control. It improves the performances of the passive system.Connected dominating set based provides the node connection. A connected dominating set based provides connection to the neighbor nodes and also provides minimum connected dominating set of graph $\mathrm{G}$ is a set $\mathrm{D}$ of vertices with two properties. The first is any node in $\mathrm{D}$ can reach any other node in $\mathrm{D}$ by a path that stays entirely within D.That is $\mathrm{D}$ indicates a connected sub graph of G.The second is every vertex in $\mathrm{G}$ either belongs to $\mathrm{D}$ or is adjacent to a vertex in D .D is the dominating set of G.Alpha minimum routing cost algorithm is used for the routing which avoids the routing traffic and provides easy transmission for the data.

\section{Algorithm}

There are two algorithms and one application used in this paper

1. Connected dominating set based algorithm

2. Alpha minimum routing algorithm

3. Semi Active state application 


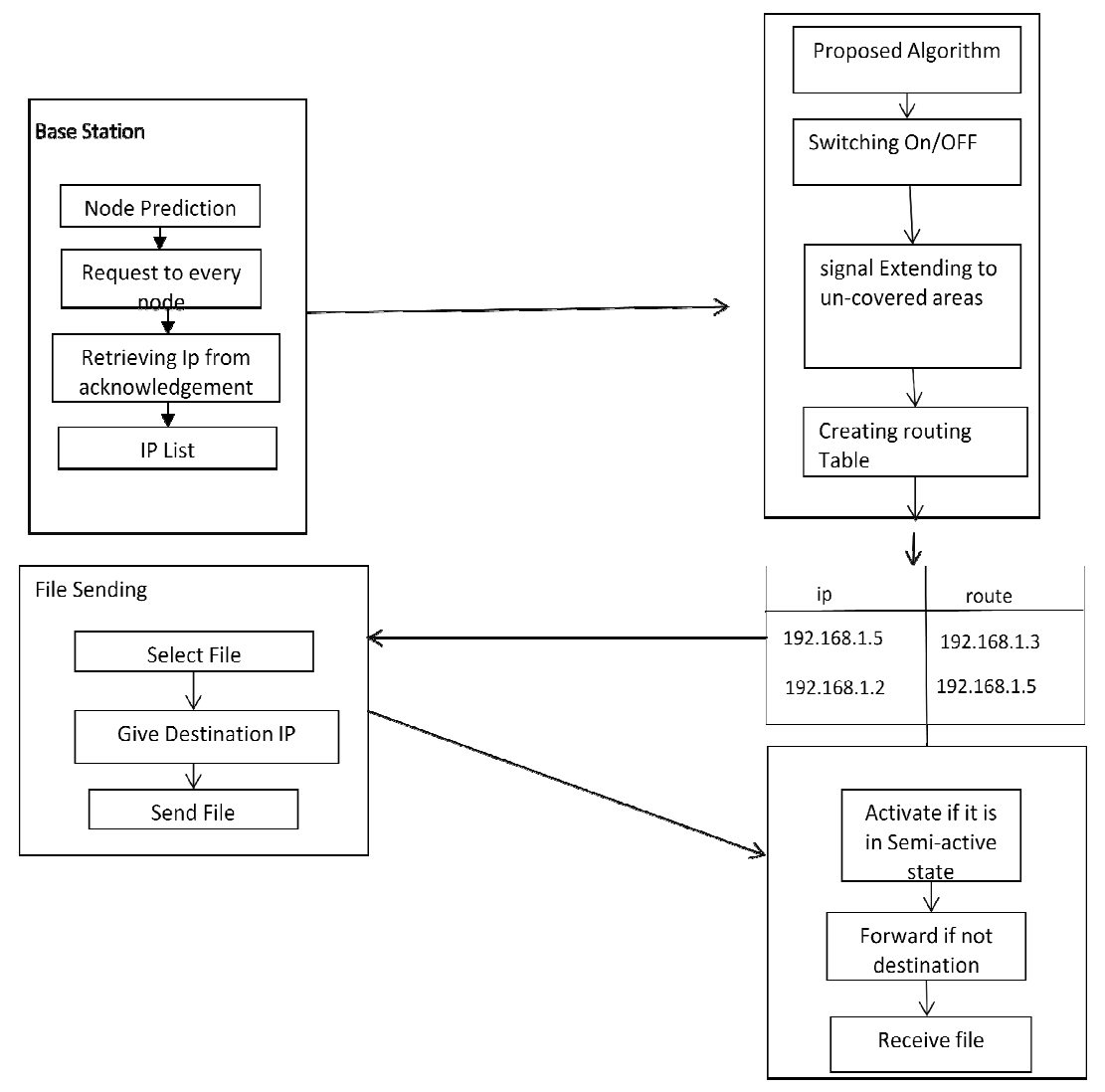

Figure 1: overall architecture

The figure 1 denotes the overall architecture of the proposed system. From the base station the node is predicted to check the neighbor nodes are available. If the nodes are within the network it sends the request to all the nodes to receive a positive acknowledgement. The acknowledgement is received based on the I address. Now the file is send to the node. The proposed algorithm is used in this stage which is shown in the fig1.Switching on and off is proposed for the nodes and the signals are extended to the uncovered areas. Routing is provided at this stage which is shown in figland an alternative path can be provided for this network if the file is not received.

\section{Routing In Wsn}

Routing plays a major role in the WSN .Routing are in different ways to reach its destination, some of the routings are location based routing, distanced based routings, hierarchalroutings, flatrouting, package based routing etc. Data are sending based on the routing. In WSN multipoint hop is done it is actually one type of ad hoc networks. WSN provides attention for the routing due to its flexibility, efficiency and scalability. Several types of attacks are produced during the routing process because it is deployed in open and unprotected environment. For critical network function nodes cannot be trusted but by processing some of the algorithm and techniques we can process it safely. In this paper we address connected dominating and alpha minimum routing algorithms for example military applications. 


\section{Sensor Network}

In wireless sensor network nodes are in the form of sensor, sensors are used to pass the data from base station to the destination sleep scheduling is provided to the sensor to save the life time of the nodes .The request is send to all the nodes to check whether the nodes are in the active or in sleep state. when the nodes are in active state the positive acknowledgment will be send to the base station or to the sender and it sends the data to the neighbor node and it hops to the next node before it hopes to next node the previous node will be in the semi active state or to the sleep node it depends upon the package. Each node wakes up independently of neighboring nodes in order to save energy. By putting nodes to sleep when there are no events, the energy consumption of the sensor nodes can be significantly reduced.

\subsection{Advantages Of Proposal}

The main advantage is to save the life time of the sensor and routing which is based on the location and the distance based. Wsn provides the efficient routing for any kind of data's or packets . The cost level is reduced by the following algorithms addressed in this paper.

\section{Application}

It can be applied to the following areas,

- Mobile phone networks

- Wifi and Wi-MAX networks

- All other wireless sensor networks

\section{Simulation Result}

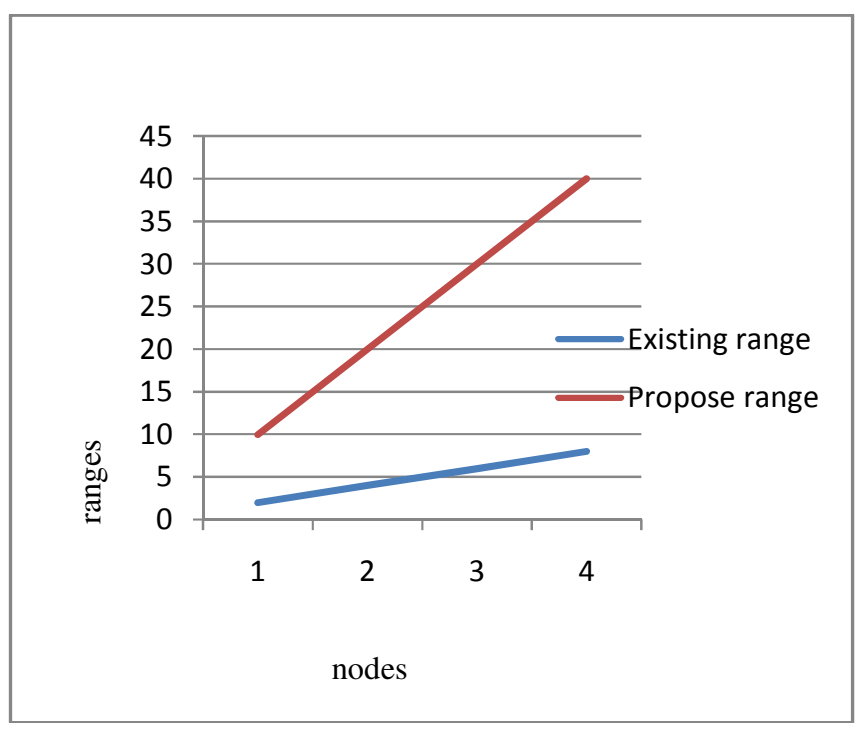

Figure 2. Lifetimes in Sensor Network 
International Journal of Control, Automation, Communication and Systems (IJCACS), Vol.1, No.1, January 2016

The Figure 2 Shows the simulation results between the existing and proposed system.

In the above figure 2 shows the comparison between the existing range and the proposed range the life time range is extended in the proposal.

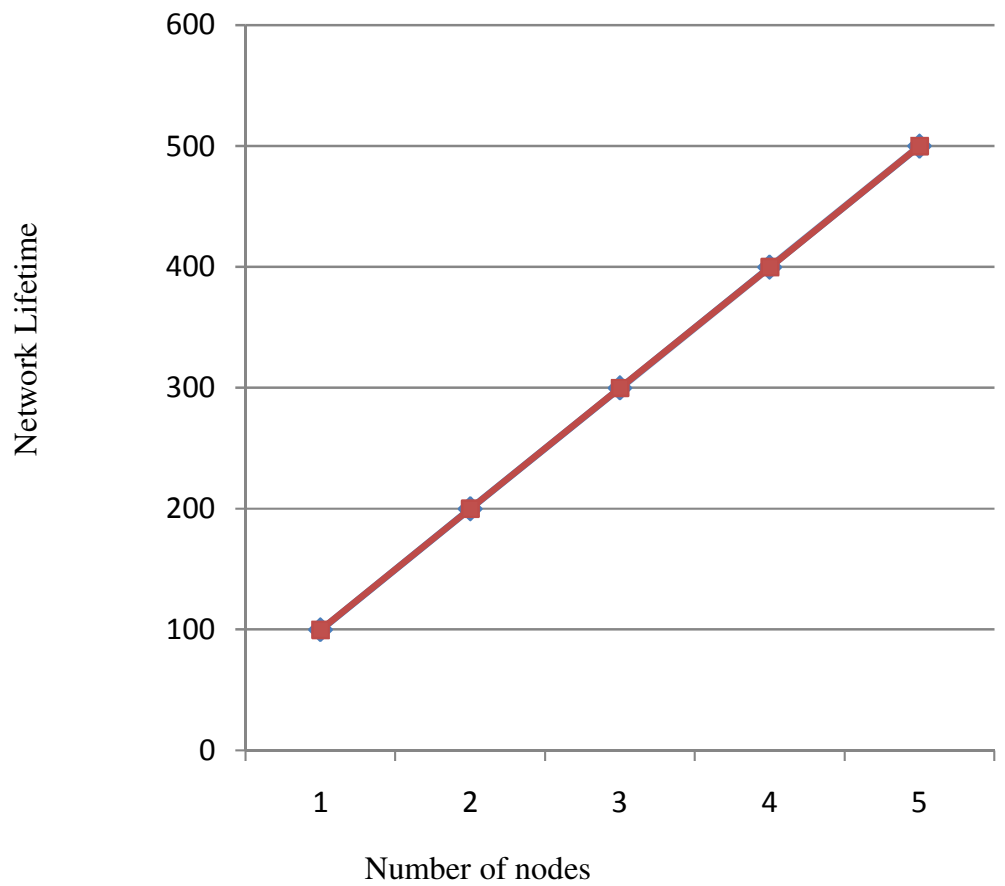

Figure 3. Lifetime Maximization in WSN

The Figure 3 shows the lifetime maximization in WSN to save the power of nodes.

The above figure shows the number of nodes and the network coverage area with the new proposal methods and techniques 


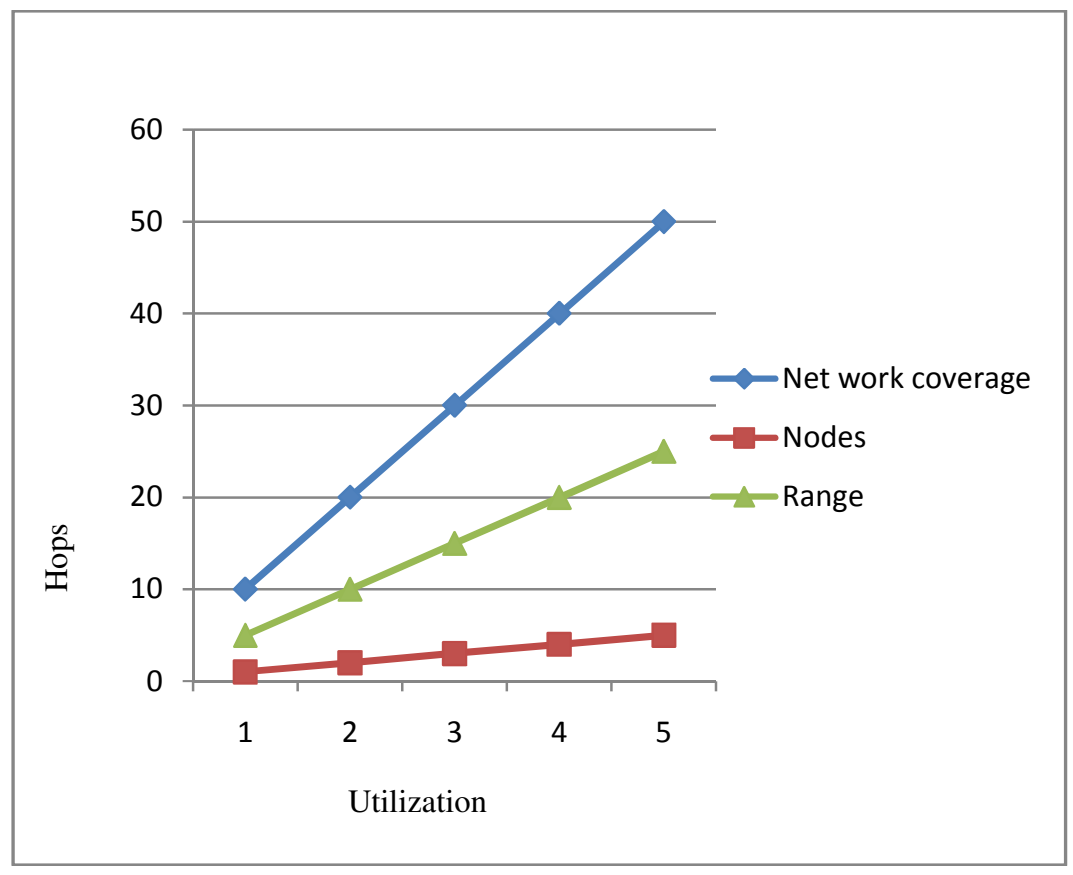

Figure4. Hops of the node and its utilization

Series 1 shows the range of the nodes

Series 2 shows the number of nodes

Series 3 shows the utilization of the network coverage

\section{CONCLUSION}

In this paper we address the life time maximization of the sensor and the routing in the WSN. The network structure is limited with these techniques such as connected dominating, alpha minimum routing cost algorithm and the sleep control by using these types we can save the life time maximization in WSN and can avoid the routing collisions and it provides signals to the uncovered area which is shown in the fig1.we can provide further more techniques to save the life time of the sensor. We also produced some highlighted techniques in this challenging network .In WSN there are many challenging works but with is proposal it is possible but it needs more techniques and algorithms to improve the life time maximization.

\section{Refernces}

[1] K.Maheswri, Dr.S.Karthik,Dr.S.KirubakaranLifetime improvement of Wireless Sensor Network by Using Sleep-WakeSchedulingand Any Cast Packet Forwarding Scheme Vol:02, 2013, pp:197-200.

[2] B. Karp and H. T. Kung, "GPSR: Greedy perimeter Stateless routing for wireless networks," in Proc.MobiCom, Vol: 01,2000,pp 243-254

[3] B. Leong, B. Liskov, and R. Morris, "Geographic routing without planarization," in Proc. NSDI, Vol: 01, 2006, pp. 339-352. 
[4] Y.-J. Kim, R. Govindan, B. Karp, and S. Shenker, "Lazy cross-link removal for geographic routing," in Proc. SenSys, 2006, pp. 112-124.

[5] L.Zhangand Y.Zhang, Energy efficient cross layer protocol of channel aware geographic-informed forwarding in wireless sensor networks," IEEE Trans. Veh. Technol., vol. 58, no. 6, pp. 3041-3052, 2009.

[6] Z.Jiang, J.Ma, W.Lou, and J.Wu, "An information model for geographic greedy forwarding in wireless ad-hoc sensor networks," in Proc. IEEE INFOCOM, 2008, pp. 825-833.

[7] Zhang and H. Shen, "Energy-efficient beaconless geographic routing in wireless sensor networks," IEEE Trans. Parallel Distrib. Syst., vol. 21, no. 6, pp. 881-896, 2010.

[8] C.-F. Hsin and M. Liu, "Network coverage using low duty-cycled sensors: Random \& coordinated sleep algorithms", in Proc.IPSN,2004 pp.433-442.

[9] P. Cheng, F. Zhang, J. Chen, Y. Sun, and X. Shen, "A distributed TDMA scheduling algorithm for target tracking in ultrasonic sensor networks," IEEE Trans. Ind. Electron., vol. 60, no. 9, pp. 3836$3845,2013$.

[10] R. C. Luo and O. Chen, "Mobile sensor node deployment and asynchronous power management for wireless sensor networks," IEEE Trans. Ind. Electron., vol. 59, no. 5, pp. 2377-2385, 2012.

[11] H. Song, V. Shin, and M. Jeon, "Mobile node localization using fusion prediction-based interacting multiple model in cricket sensor network," IEEE Trans. Ind. Electron., vol. 59, no. 11, pp. 43494359, 2012.

[12] J. Pan, L. Cai, Y. T. Hou, Y. Shi, and X. X. Shen, "Optimal base-station locations in two-tiered wireless sensor networks,” IEEE Trans. Mobile Comput., vol. 4, no. 5, pp. 458-473,2005. 